\title{
Kyphomelic dysplasia in two sib fetuses
}

\author{
Chih-Ping Chen, Schu-Rern Chern, Shin-Lin Shih, Chun-Yu Chuang, Fu-Yuan Huang
}

\section{Department of \\ Obstetrics and \\ Gynaecology, Mackay \\ Memorial Hospital, 92, Section 2, Chung-Shan North Road, Taipei, Taiwan, Republic of China \\ C-P Chen}

\section{Department of Medical Research, Mackay Memorial Hospital, 92, Section 2, Chung-Shan North Road, Taipei, Taiwan, Republic of China C-P Chen S-R Chern C-Y Chuang F-Y Huang}

\section{Department of} Radiology, Mackay Memorial Hospital, 92, Section 2, Chung-Shan North Road, Taipei, Taiwan, Republic of China

S-L Shih

\section{Department of} Paediatrics, Mackay Memorial Hospital, 92, Section 2, Chung-Shan North Road, Taipei,

Taiwan, Republic of China

F-Y Huang

Correspondence to: Dr Chen.

Received 21 April 1997 Revised version accepted for publication 1 July 1997

\begin{abstract}
We present the in utero appearances and postmortem radiographic findings of two sib fetuses, a male and a female, with features suggestive of kyphomelic dysplasia. The fetuses had severe bowing of the long bones, short, flared ribs, platyspondyly, metaphyseal flaring, skin dimpling, with normal external genitalia and karyotypes and a normal pregnancy. They were born to a mother with features of brachydactyly type E. Prenatal ultrasonography of each case showed a normal amount of amniotic fluid, a normal brain, a normal biparietal diameter, symmetrical bowing and shortening of the long bones, and a narrow thorax. Our cases provide support for a familial mode of inheritance for both sexes in kyphomelic dysplasia. Prenatal ultrasound examination can be offered in subsequent pregnancies.

(F Med Genet 1998;35:65-69)
\end{abstract}

Keywords: kyphomelic dysplasia; prenatal diagnosis ultrasound; familial inheritance

Kyphomelic dysplasia is a rare, congenital, skeletal disorder characterised by a short trunk, micrognathia, midfacial hypoplasia, a long philtrum, a normal occipitofrontal circumference, severe rhizomelic/mesomelic limb shortening, platyspondyly, irregular, flared metaphyses, bowing of the limbs, skin dimpling over bowing, short, angulated femora, a narrow chest, and joint restriction. ${ }^{1}$ Khajavi et al first reported two male cases in 1976 as a variant normocephalic type of short limbed campomelic syndrome. In 1979, Hall and Spranger ${ }^{3}$ further described one pair of affected brothers with congenital bowing and short, broad bones. In 1983, Maclean et al proposed the nomenclature "kyphomelic dysplasia" to describe the skeletal dysplasia in a male infant with short, angulated femora and bowing of other long bones and the cases reported by Khajavi et $a l^{2}$ and Hall and Spranger. ${ }^{3}$ Since then, 13 cases of kyphomelic dysplasia have been reported..$^{2-11}$ None of these reports included the appearance in utero. We report two sib fetuses, a female and a male, with kyphomelic dysplasia and distinctive prenatal findings.

\section{Case reports}

CASE 1

During her first pregnancy, a 25 year old primigravida came to our hospital for antenatal care from 17 weeks' gestation. Her height was $150 \mathrm{~cm}$. She had acromelic shortening of the fingers and toes, especially the thumbs, little fingers, and big toes, hypoplastic nails, isolated, and short 4 th metatarsals with the 4 th toes inserting into the feet, which were consistent with the diagnosis of brachydactyly type E. She and her spouse were Chinese and nonconsanguineous. She had neither prenatal exposure to teratogenic drugs nor a family history of malformations. Maternal urine throughout the pregnancy did not contain glucose. Radiographic examination showed shortened metacarpals and phalanges of the hands, shortened metatarsals and phalanges of the feet, 12 pairs of ribs, and normal scapulae, clavicles, humeri, femora, ischial bones, and tibiae. She had a normal karyotype, 46,XX.

Prenatal ultrasonography at 26 weeks' gestation showed a normal amount of amniotic fluid, a biparietal diameter of $67 \mathrm{~mm}$, equal to 26 weeks' gestation, symmetrical angulation and shortening of the long bones, normal cerebral ventricles, and a narrow chest. The long bone lengths measured below the 5 th centile, with the femur measuring $36 \mathrm{~mm}$, humerus 32 $\mathrm{mm}$, and tibia $30 \mathrm{~mm}$ (fig 1). Amniocentesis showed a 46,XX karyotype. The mother decided to terminate the pregnancy at 31 weeks' gestation. The dead infant measured $34.5 \mathrm{~cm}$ in length and weighed $1000 \mathrm{~g}$. Physical examination showed short limbs, joint restriction, a normal occipitofrontal circumference, a normal palate, a flat nasal bridge, hypertelorism, a long philtrum, low set ears, a narrow thorax, pretibial skin dimpling, normal hands and feet, and normal female external genitalia (fig 2). Postmortem radiographic examination (fig 3) showed symmetrical, short long bones with bowed femora, humeri, tibiae, fibulae, ulnae, and radii, 12 pairs of short, flared ribs, normal clavicles and scapulae, a narrow thorax, platyspondyly, metaphyseal flaring, and ossification of the tali. The dysmorphic features and radiological findings (fig 1) of the bowed long bones removed from the proband were consistent with the diagnosis of kyphomelic dysplasia. The internal organs were normal at necropsy.

CASE 2

During her third pregnancy, the same mother and father as of case 1 came for prenatal care six years after the delivery of their first malformed child. Five years previously, the mother had given birth to her second child, a healthy boy without any skeletal abnormalities. Prenatal ultrasonogrphy at 23 weeks' gestation showed a normal amount of amniotic fluid, a biparietal diameter of $56.3 \mathrm{~mm}$, equal to 23 weeks' gestation, normal cerebral ventricles, a narrow chest, and symmetrical angulation and shortening of the long bones. The long bones measured below the 5th centile, with the femur measuring $25.8 \mathrm{~mm}$, tibia $23.8 \mathrm{~mm}$, fibula 23.6 $\mathrm{mm}$, humerus $29.1 \mathrm{~mm}$, ulna $23.6 \mathrm{~mm}$, and 


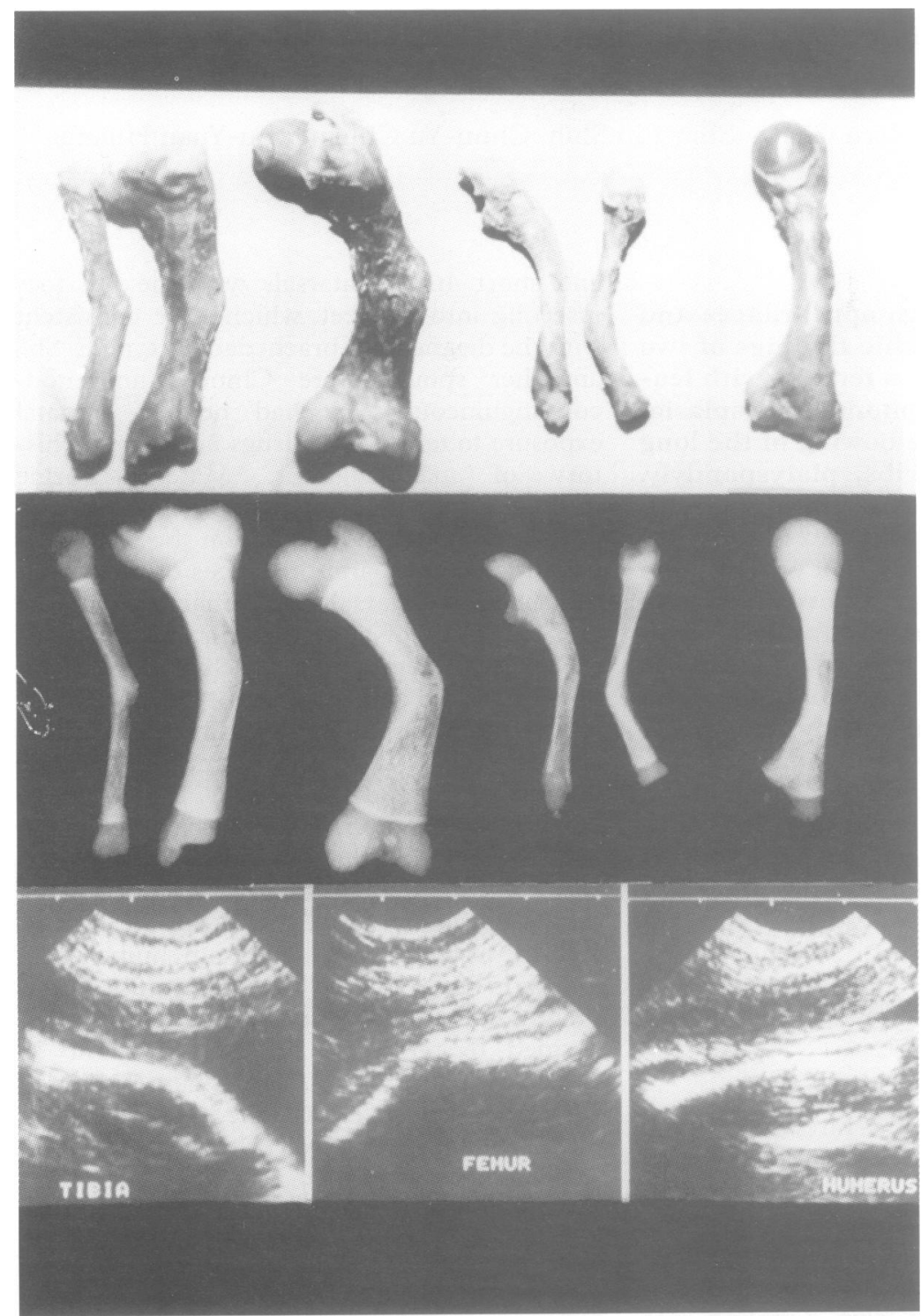

Figure 1 The clinical and radiographic appearance of the long bones removed from case 1 (order from left to right: fibula, tibia, femur, radius, ulna, and humerus). Prenatal ultrasonography at 26 weeks' gestation showing in utero angulation of the tibia, fibula, and humerus.

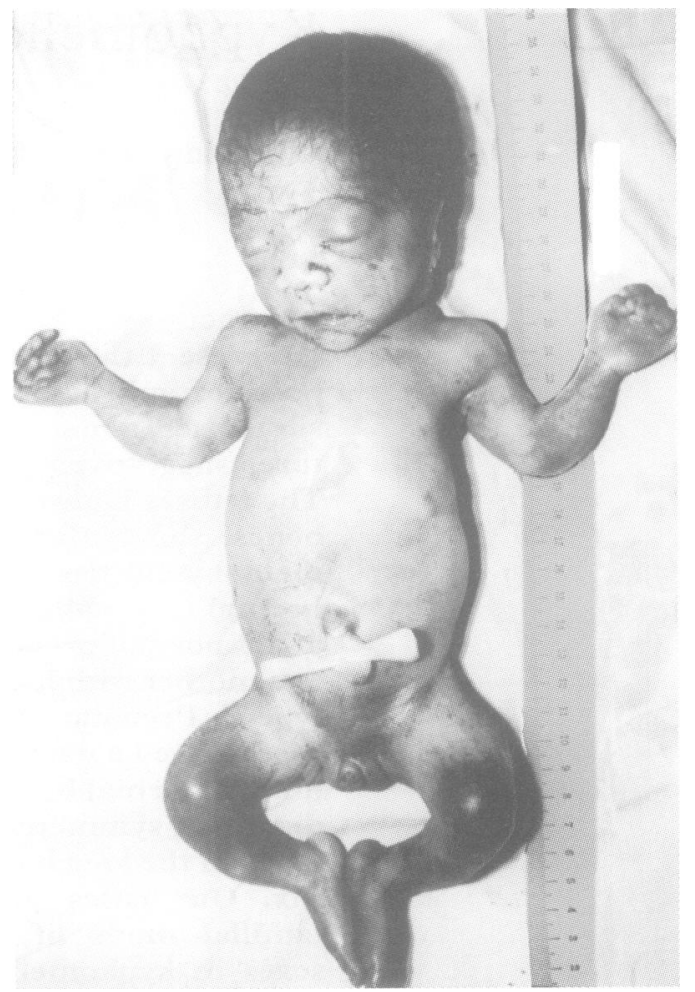

Figure 2 Case 1 at birth.

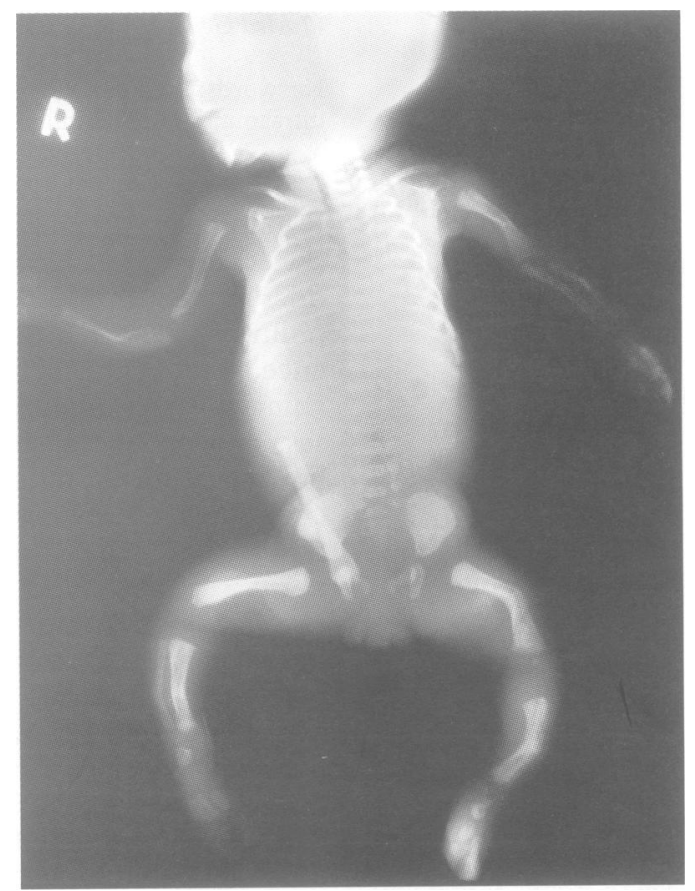

Figure 3 Whole body radiograph of case 1.

radius $23.8 \mathrm{~mm}$ (fig 4). Amniocentesis showed a 46,XY karyotype. The same findings as in the earlier case were obvious. The mother opted to terminate the pregnancy at 23 weeks' gestation. The infant at birth weighed $600 \mathrm{~g}$ and measured $26 \mathrm{~cm}$ in length. Physical examination showed a normal occipitofrontal circumference, a normal palate, a flat nasal bridge, hypertelorism, a long philtrum, low set ears, short limbs, joint restriction, a narrow thorax, pretibial skin dimpling, normal hands and feet, and normal male external genitalia (fig 5). Postmortem radiographic examination (fig 6) showed short long bones with symmetrically bowed femora, humeri, tibiae, fibulae, radii, and ulnae, 12 pairs of short, flared ribs, normal clavicles and scapulae, platyspondyly, metaphyseal flaring, and ossification of the tali.

Samples of DNA were obtained from parental whole blood, umbilical cord, and muscle of case 2. Polymerase chain reaction methods using primers flanking the SOX9 gene were applied according to previously published methods. ${ }^{12}{ }^{13}$ The amplification was carried out

in an "OmniGene" module (HYBAID, UK) with $30-35$ cycles at $94^{\circ} \mathrm{C}$ for 60 seconds, $55-64^{\circ} \mathrm{C}$ for 60 seconds, and $72^{\circ} \mathrm{C}$ for two minutes. Sequencing reactions were performed with the ABI PRISM dye terminator cycle sequencing kit (Perkin Elmer, USA) and then analysed on an ABI 377 automated DNA sequencer. Both strands were sequenced using either the forward or reverse primer. Paternity was confirmed by a DNA fingerprint of the family. Analysis of the SOX9 gene in case 2 showed no mutation in either of the SOX9 alleles. The internal organs were normal at necropsy. 

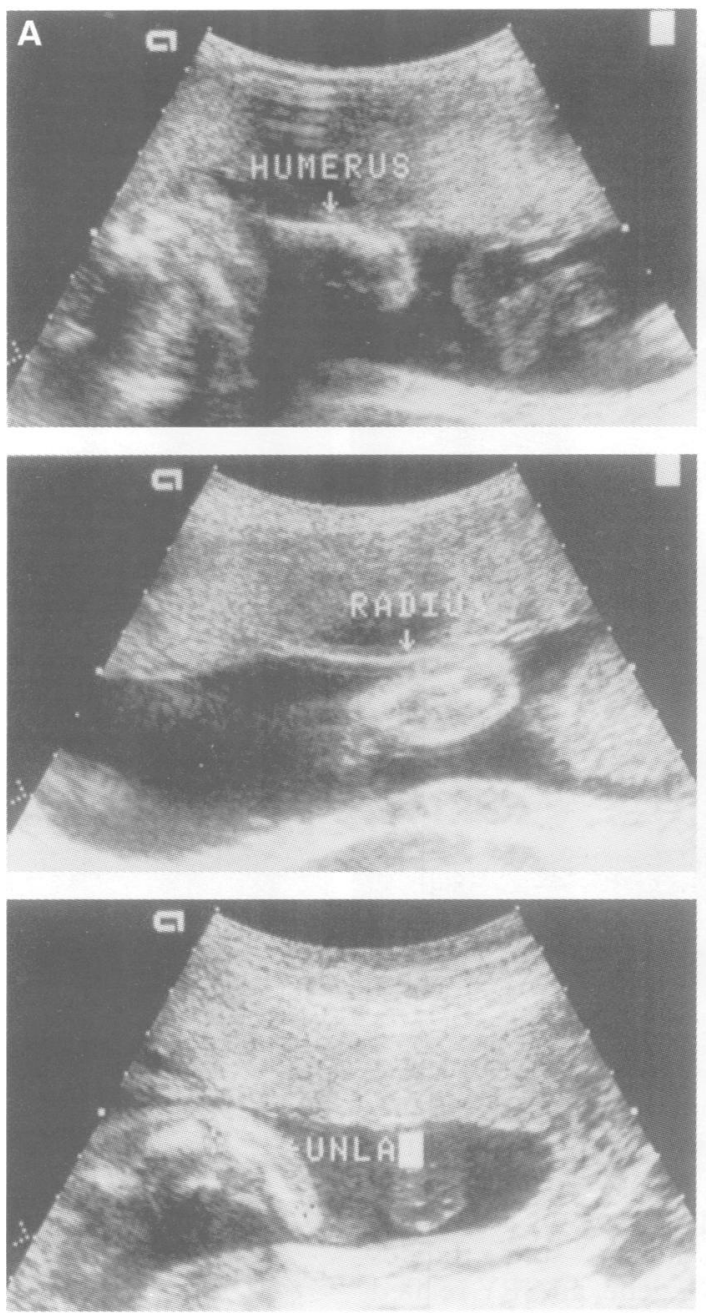

Figure 4 Prenatal ultrasonography of case 2 at 23 weeks' gestation showing in utero angulation of $(A)$ the humerus, radius, and ulna, (B) the femur, tibia, and fibula.

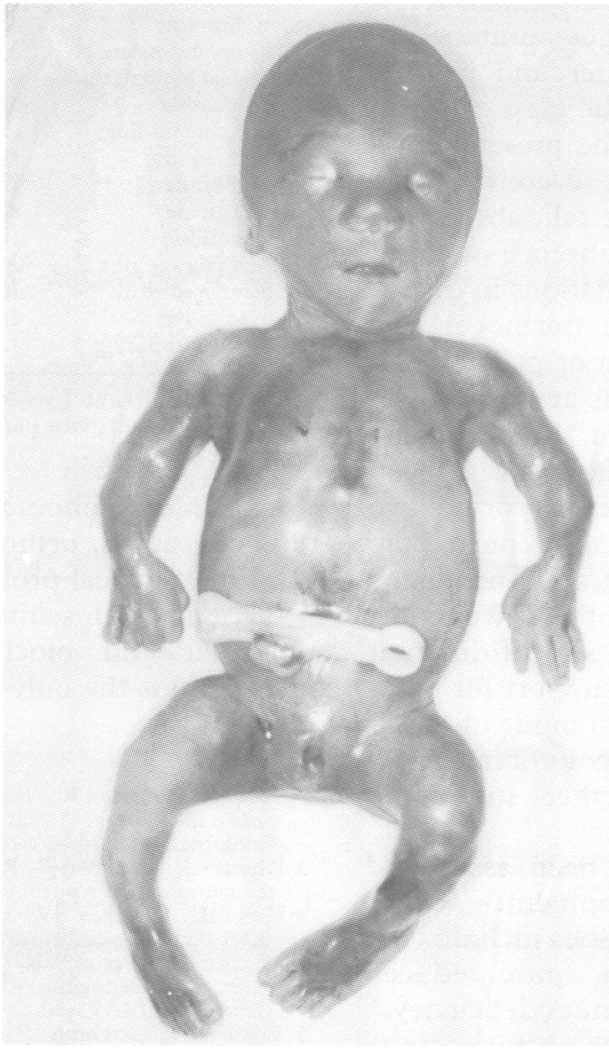

Figure 5 Case 2 at birth.
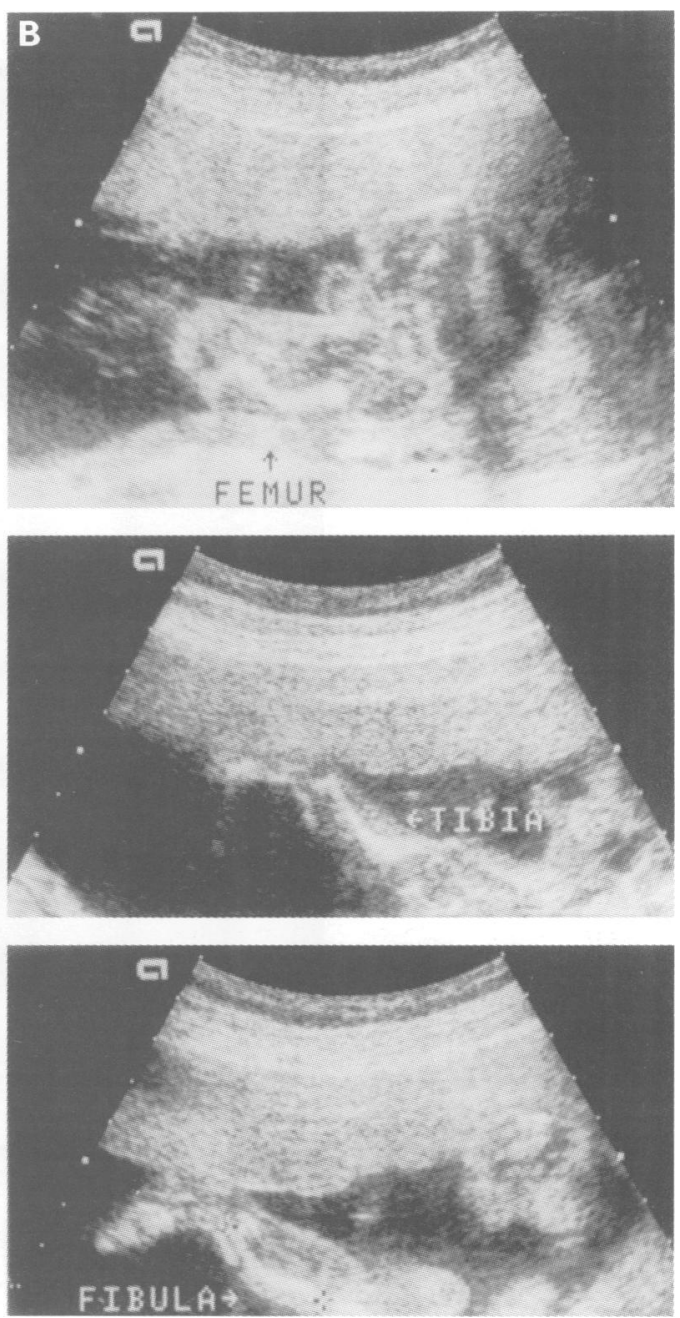

\section{Discussion}

A comparison of our probands with the 13 previously reported cases of kyphomelic dysplasia is shown in table 1. Differential diagnosis of kyphomelic dysplasia should include short stature/short limb skeletal dysplasia (SLSD) with severe combined immunodeficiency (SCID), campomelic dysplasia, congenital bowing of the long bones, Antley-Bixler syndrome, Larsen syndrome, diastrophic dysplasia, Stüve-Wiedemann dysplasia, Fuhrmann syndrome, and asymmetrical (symmetrical) long bone bowing with craniosynostosis. ${ }^{1}$ Short stature/SLSD with SCID is characterised by autosomal recessive inheritance, bowing of the long bones, metaphyseal chondrodysplasia, flaring of the rib ends, decrease in the vertical height of vertebral bodies, lymphopenia, reduced immunoglobulin levels, recurrent respiratory infection, diarrhoea and moniliasis. ${ }^{14}$ The classical form of campomelic dysplasia is characterised by autosomal dominant inheritance, sex reversed XY females, dwarfism, respiratory distress, macrocephaly, micrognathia, malformed ears, cleft palate, bowing of the lower limbs, central nervous system anomalies, neonatal death, polyhydramnios, associated abnormalities such as hydronephrosis and congenital heart defects, an enlarged and elongated skull, hypoplasia of the cervical vertebrae, a bell shaped thorax with 

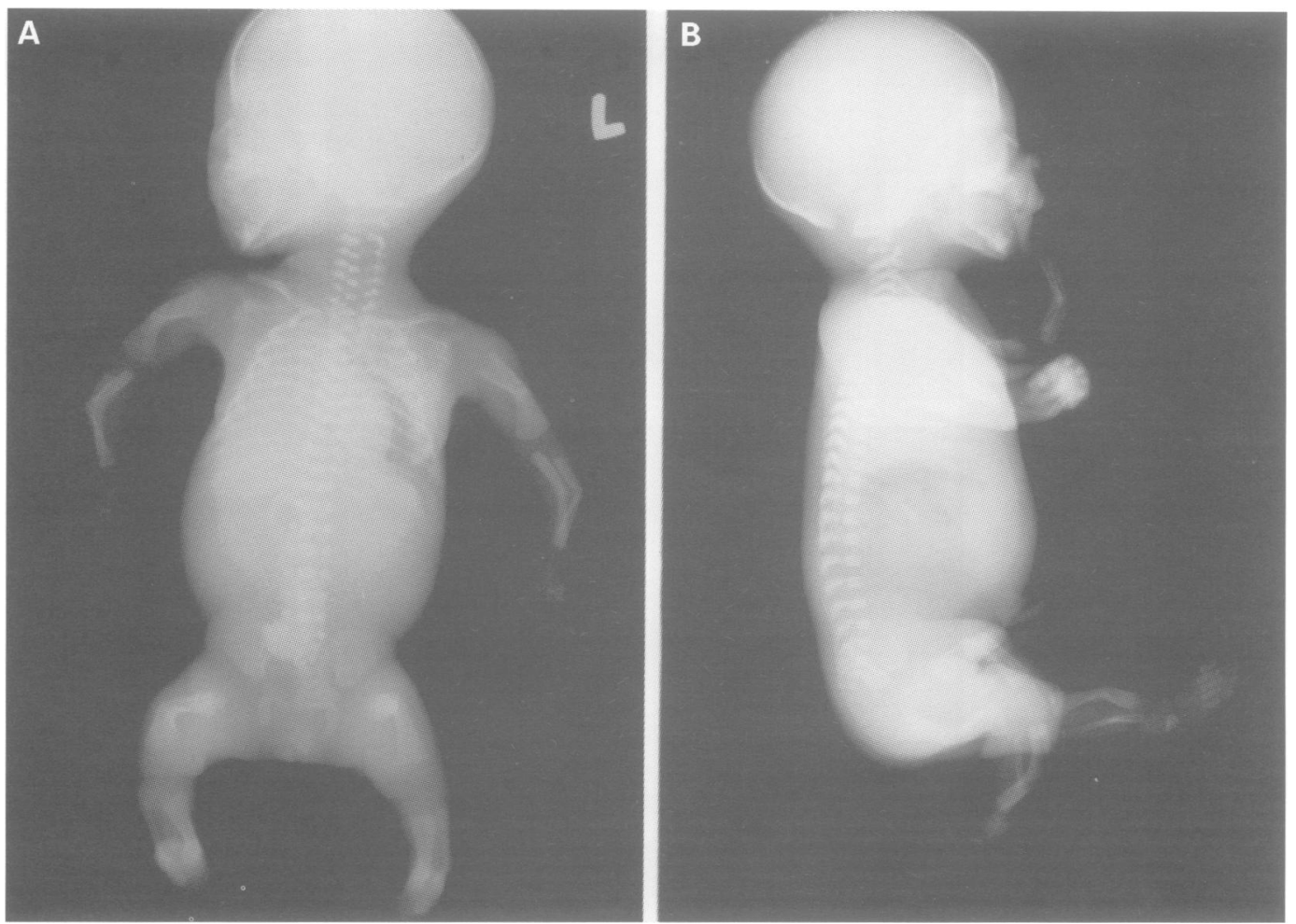

Figure 6 (A) Anteroposterior and (B) lateral views of the whole body radiographs of case 2.

11 pairs of ribs, hypoplastic scapulae, some shortness and angulation of the upper extremities, a contracted pelvis, angulation of the proximal femoral shaft, hypoplasia and angulation of the tibia, hypoplasia of the fibula, no ossification of the talus, and talipes equinovarus. ${ }^{1}$ The SOX9 gene, an SRY related gene located on chromosome 17q24.3-q25.1, has been considered a candidate gene for sex reversal and campomelic dysplasia. ${ }^{12} 131516$ Campomelic dysplasia is an autosomal dominant disorder with a heterozygous mutation in SOX9. ${ }^{12} 1316$ The present sister and brother pair was distinguished from the classical form of campomelic dysplasia by the presence of a normal cranium and brain, severely bowed extremities, ossification of the tali, absence of hypoplasia of the fibula, and absence of either sex reversal or SOX 9 gene mutations in case 2 . Some reported cases of the normocephalic form of campomelic dysplasia or pseudocampomelia have been regarded as actual cases of kyphomelic dysplasia. ${ }^{1}$ To date, three sib pairs with kyphomelic dysplasia have been described. ${ }^{1}$ Hall and Spranger ${ }^{3}$ reported two affected brothers, Rezza et $a l^{11}$ reported an affected brother and sister pair, and Turnpenny et al reported two affected brothers. We report an additional pair of affected sibs of different sexes which provides further support for autosomal inheritance. The familial mode of inheritance may be either homogeneous or heterogeneous and will require further studies to elucidate this.

Kyphomelic dysplasia has been associated with normal mental development. ${ }^{17}$ Other associated reported abnormalities include cleft lip, cleft palate, and apnoea, ${ }^{6}$ pure red cell aplasia, ${ }^{7}$ severe combined immunodeficiency, ${ }^{10}$ Perthes disease, ${ }^{9}$ and avascular necrosis of the proximal femoral epiphyses. ${ }^{8}$ Although the
Table 1 Clinical and radiographic findings of the present two cases and the 13 previously reported cases ${ }^{\star}$

\begin{tabular}{llll}
\hline & $\begin{array}{l}\text { Previous } \\
\text { reportst } \\
(13 \text { cases })\end{array}$ & \multicolumn{2}{c}{ Present reports } \\
\cline { 4 - 4 } \cline { 3 - 4 } & & Case 1 & Case 2 \\
\hline Clinical findings & $10 \mathrm{M} / 1 \mathrm{~F}$ & $\mathrm{~F}$ & $\mathrm{M}$ \\
Sex & $11 / 11$ & Yes & Yes \\
Normal OFC & $10 / 10$ & Yes & Yes \\
Small chest & $12 / 12$ & Yes & Yes \\
Short limbs & $5 / 5$ & Yes & Yes \\
Limited joint movement & $5 / 9$ & Yes & Yes \\
Normal hands and feet & $6 / 6$ & Yes & Yes \\
Skin dimpling & $2 / 12$ & No & No \\
Cleft lip and palate & $6 / 8$ & Yes & Yes \\
Midfacial hypoplasia & $5 / 8$ & No & No \\
Micrognathia & & & \\
Radiographic findings & $13 / 13$ & Yes & Yes \\
Bowed femora & $7 / 12$ & Yes & Yes \\
Bowed humeri & $8 / 11$ & Yes & Yes \\
Bowed tibiae & $10 / 12$ & Yes & Yes \\
Bowed radii & $2 / 10$ & No & No \\
Eleven pairs of ribs & $10 / 10$ & Yes & Yes \\
Short, flared ribs & $4 / 9$ & Yes & Yes \\
Platyspondyly & $11 / 11$ & Yes & Yes \\
Metaphyseal flaring & & &
\end{tabular}

*Data compiled from references $2-11$

†Expressed only in those patients with specific findings well described in reports.

prognosis of kyphomelic dysplasia appears to be encouraging, orthopaedic sequelae as well as haematological problems should be advised in genetic counselling. In the absence of molecular and biochemical tests, prenatal ultrasound is the only tool available for prenatal diagnosis.

1 Taybi H, Lachman RS. Radiology of syndromes, metabolic disTaybi H, Lachman RS. Radiology of syndromes, metabolic dis-
orders, and skeletal dysplasia. 4th ed. St Louis: Mosby-Year orders, and skeletal dysplasia. 4th ed.

2 Khajavi A, Lachman R, Rimoin D, et al. Heterogeneity in the campomelic syndromes. Radiology 1976;120:641-7.

3 Hall BD, Spranger JW. Familial congenital bowing with short bones. Radiology 1979;132:611-14.

4 Maclean RN, Prater WK, Lozzio CB. Skeletal dysplasia with short, angulated femora (kyphomelic dysplasia). $\mathrm{Am} \mathcal{F} \mathrm{Med}$ Genet 1983;14:373-80

5 Viljöen D, Beighton P. Kyphomelic dysplasia: further delineation of the phenotype. Dysmorphol Clin Genet 1988; 1:136-41. 
6 Temple IK, Thompson EM, Hall CM, Bridgeman G, Pembrey ME. Kyphomelic dysplasia. $\mathcal{F}$ Med Genet 1989;26:45761 .

7 Turnpenny PD, Dakwar RA, Boulos FN. Kyphomelic dysplasia: the first 10 cases. $\mathcal{F}$ Med Genet 1990;27:269-72. 8 Maffulli N, Blakeway C, Fixsen JA. Long-term follow up of a case of kyphomelic dysplasia. Orthopedics 1993;16:86-9. 9 O'Reilly MAR, Hall CM. Kyphomelic dysplasia. $\mathcal{f} \mathrm{Med}$ Genet 1994;31:423.

10 Corder WT, Hummel M, Miller C, Wilson NW. Association of kyphomelic dysplasia with severe combined immunodeof kyphomelic dysplasia with severe combin

11 Rezza E, Iannacconne G, Lendvai D. Familial congenital Rezza E, lannacconne $G$, Lendvai $D$. Familial congenital
bowing with short thick bones and metaphyseal changes, a distinct entity. Ped

12 Wagner $\mathrm{T}$, Wirth $\mathrm{J}$, Meyer J, et al. Autosomal sex reversal and campomelic dysplasia are caused by mutations in an around the SRY-related gene SOX9. Cell 1994;79:1111-20.
13 Kwok C, Weller PA, Guioli S, et al. Mutations in SOX9, the gene responsible for campomelic dysplasia and autosomal sex reversal. Am f Hum Genet 1995;57:1028-36.

14 MacDermot KD, Winter RM, Wigglesworth JS, Strobel S Short stature/short limb skeletal dysplasia with severe combined immunodeficiency and bowing of the femora: report of two patients and review. F Med Genet 1991;28:10-17.

15 Wright E, Hargrave MR, Christiansen J, et al. The Sry-related gene SOX 9 is expressed during chondrogenesis Sry-related gene $S O X 9$ is expressed during
in mouse embryos. Nat Genet 1995;9:15-20.

16 Foster JW, Dominguez-Steglich MA, Guioli S, et al. Faster JW, Dominguez-Steglich MA, Guioli $S$, et al. by mutations in an SRY-related gene. Nature 1994;372: b25-30.

17 McKusick VA. 211350. Bowing, congenital, with short bones [kyphomelic dysplasia]. In: McKusick VA, ed. Mendelian inheritance in man. 10th ed. Baltimore: Johns Hopkins University Press, 1992:1257. 